\title{
Foreword
}

\section{Belief Expression of the Chairperson Assumption of the IEIJ}

\author{
Hisanori TOYOSHIMA
}

Hitachi Global Life Solutions, Inc.

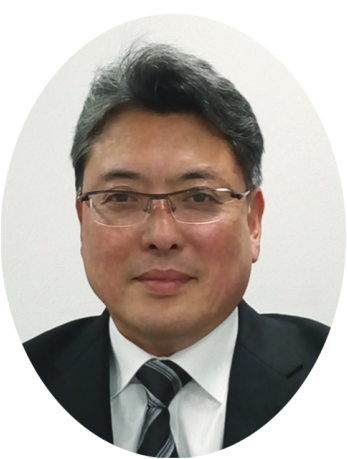

This Text is an excerpt from the journal of the IEIJ, Vol. 103 No. 9, 2019.

At the recommendation of the members, I have been appointed as Chairperson of the Illuminating Engineering Institute of Japan (IEIJ). I am honored to undertake this important role at the outset of the new Reiwa era, and at the same time, I have renewed my determination in the face of this heavy responsibility.

As an academic society with 103 years of history, I imagine that the IEIJ has worked to change itself in line with the needs of the times in enduring over the course of a century. I would like to express my esteem for former Chairperson Inoue and the other senior members who have contributed greatly to the management of the IEIJ thus far. And I would also like to convey my intent to steadfastly execute the initiatives that have been advanced until now, as well as to explore new approaches to business management that address the needs of the times.

Looking back over the standing of the IEIJ in recent years, on the occasion of its 100th anniversary in 2016, the IEIJ formulated its Master Plan (medium-term vision 2016-2021) based on its long-term vision (Grand Design), and has advanced initiatives designed to deepen its functions and to strengthen its foundation as an academic society. As one example, in June 2019 the IEIJ worked to consolidate its expert groups with overlapping duties (from 8 to 4) and established a comprehensive subcommittee management framework. The IEIJ has also been undertaking initiatives like establishing a "Lighting Professional" program designed to provide human resources support and strengthen its presence among engineers.

As part of its business plan for fiscal 2019, the IEIJ will work to revitalize its activities in accordance with the vision mentioned above and optimize its business management. I will do my best to pursue my duties in order to steadfastly execute initiatives that focus on enhancing the IEIJ functions, human resource development, and information dissemination. At the same time, however, I know there will be many areas in which I will require the support of the members, and would thus like to ask for your continued support.

In terms of recent transformations within the industry, in April 2019 the Japanese National Committee of CIE (JCIE) merged with the Japan Lighting Manufacturers Association (JLMA). Even now the IEIJ and the JLMA continue to cooperate on JIS/IEC standardization activities. Likewise, I intend to further strengthen collaboration, including serving as the contact point for CIE in Japan, and to contribute to the further development of optical and lighting sciences in Japan and around the world.

In recent years, the environment encompassing us has been in a constant state of change. As put forth in the IEIJ's long-term vision, it goes without saying that, "while addressing the changes in Japan and the world, the intent to taking the lead in the study of lighting as the symbol of civilization and also in the investigation and research related to its application" will be an essential aspect of future research activities and business. In addition, initiatives targeting the goals of the rapidly spreading concept of "Contributing to the sustainable development of mankind and society," in short, the Sustainable Development Goals (SDGs), will be also important goals for the IEIJ in addressing the changes faced by Japan and the world. In the current market, remarkable progress has been made in the shift to Solid State Lighting (SSL) as a light source for standard lighting, and we can imagine that the shift to SSL in the housing stock market will have a considerable impact on the economy and climate in terms of energy savings. In recent years, many members have been working to improve academia and technology in various ways. In some cases, these include different concepts of connectivity for terminal devices as part of IoT-based system technologies, while in others these include accelerating research and development into new light sources, such as OLEDs and lasers. More recently, these have included cross-field application examples that link the lighting field with other areas, 
like health, medicine, and agriculture. In this way, I am confident that the optical and lighting sciences, particularly because they go beyond simple "illumination" to play a role in many other scenarios and applications, will enable us to approach many of these goals.

As I mentioned a moment ago about the shift to SSL in standard lighting, in general, markets that are saturated with products based on fairly established technologies tend to see differences in quality and function emerge in response to consumer preferences. In terms of options for "high added value" based on high quality and additional functions, we can imagine lighting being equipped with advanced technologies and previously unseen functions or those that have been adopted by other markets. When we look at this from the perspective of lighting equipment, we have also seen the commercialization of products that apply the concept of circadian rhythms, for example, and products that consider lighting equipment as a type of IoT device that is hung from the ceiling. This trend indicates the potential for existing definitions and concepts of "lighting" to become transformed as part of market trials. And along with serving as evidence of advancement in the development and application of scientific and manufacturing technology, we can assume that this trend will require the IEIJ to take on an increasing number of essential roles, including the promotion of technology testing, application, assessment, and standardization, where scientific assessment of definitions and results will become an important perspective. I also regard these as one of the changes in society and will thus work to support academic and practical approaches to various issues. I hope that all members actively participate in these various activities.

Although the IEIJ is only just beginning its activities for the next 100 years, including those to address these changes, I believe the most important approach to further enhancing its activities is nurturing the human resources who will lead the next generation. I intend to continue promoting certification of qualified lighting engineers and lighting consultants, expanding distance learning programs, and holding the Young Wave Forum, as well as providing opportunities for young researchers and corporate practitioners to present their research. Based on the trends in the lighting industry, I believe that strengthening cooperation with researchers and business practitioners from different industries and academic fields will be an extremely important issue for the IEIJ and the industry. I will therefore undertake initiatives to strengthen information dissemination and to increase the presence of the IEIJ.

Finally, I also recognize the need to strengthen the foundation of the IEIJ management in order to support the activities of its members.

In order to provide opportunities for the development, growth, support, and encouragement of the members through these IEIJ activities, I would like to once again ask for the further understanding and cooperation of everyone. 